\title{
DÉFORMATION DE L'ALGÈBRE DES COURANTS ASSOCIÉE AU GROUPE DE POINCARÉ
}

\author{
FAOUZI AMmAR
}

Abstract

Our main purpose in this paper is to compute the second group of local cohomology of the current Lie algebra $\mathbb{G}_{F}$ with type Poincaré Lie group $G$ and stated the local deformations associated.

\section{Introduction}

1.1. L'algèbre des courants. On considère $F$ un fibré principal sur une variété $M$ de groupe de structure $G$ dont l'algèbre de Lie est $\mathbb{G}$. On note $F_{\mathbb{G}}$ le fibré vectoriel associé à $F$ correspondant à la représentation adjointe de $G$ dans $\mathbb{G}$. L'espace des applications $\mathbb{G}$-équivariantes de l'espace total de $F_{\mathbb{G}}$ à valeurs dans $\mathbb{G}$ s'identifie à l'espace des sections du fibré vectoriel $F_{\mathbb{G}}$, noté $\mathbb{G}_{F}$.

On a ainsi sur $\mathbb{G}_{F}$ une estructure d'algèbre de Lie donnée point par soint par celle de $\mathbb{G}$. L'algèbre de Lie $\mathbb{G}_{F}$ est appelée l'algèbre des courants du fibré principal $F$. On rappelle qu'une $p$-cochaine $c$ de $\mathbb{G}_{F}$ dans $\mathbb{G}_{F}$ est locale si $\operatorname{supp} c\left(s_{1}, \ldots, s_{p}\right) \subseteq \bigcap_{1 \leq i \leq p} \operatorname{supp} s_{i}$ pour tous $s_{1}, \ldots, s_{p} \in \mathbb{G}_{F}$ et supp désigne le support d'une section. On vérifie facilement que l'espace des cochaines locales de $\mathbb{G}_{F}$ dans $\mathbb{G}_{F}$ forme un sous complexe du complexe de Chevalley-Eilenberg de $\mathbb{G}_{F}$ à coefficients dans la représentation adjointe et sa cohomologie est notée $H_{\text {loc }}^{*}\left(\mathbb{G}_{F}, \mathbb{G}_{F}\right)$.

1.2. L'algèbre de Lie $\mathbb{G}_{\infty}(\boldsymbol{m})$. Si $m=\operatorname{dim} M$, on note $\mathbb{G}_{\infty}(m)$ l'algèbre de Lie des séries formelles à coefficients dans $\mathbb{G}$ et en $m$ indéterminées $t_{1}, \ldots, t_{m}$. Pour un multi indice $\alpha=\left(\alpha_{1}, \ldots, \alpha_{m}\right)$ et pour $x_{\alpha} \in \mathbb{G}$ on note:

$x_{\alpha} t^{\alpha}=x_{\alpha} t_{1}^{\alpha_{1}} \ldots t_{m}^{\alpha_{m}}$. Les éléments de $\mathbb{G}_{\infty}(m)$ s'écrivent sous la forme $\sum_{\alpha} t^{\alpha} x_{\alpha}$. On note $|\alpha|$ la longueur de $\alpha$, soit $|\alpha|=\sum_{i=1}^{m} \alpha_{i}$. 
L'algèbre de Lie $\mathbb{G}_{\infty}(m)$ opère naturellement sur $\mathbb{G}$ par l'action adjointe du terme constant, soit $\sum_{\alpha} t^{\alpha} x_{\alpha} \rightarrow$ ad $x_{0}$.

Soit $c$ une $p$-cochaine de $\mathbb{G}_{\infty}(m)$ dans $\mathbb{G}$, pour tout multi indice $\alpha$ on définit la composante de $c$ notée $c_{\alpha}$ comme étant l'application linéaire de $\mathbb{G}^{p}$ dans $\mathbb{G}$ définie par:

$$
\begin{aligned}
& c_{\alpha}\left(x_{1}, \ldots, x_{p}\right)=c\left(x_{1} t^{\alpha_{1}}, \ldots, x_{p} t^{\alpha_{p}}\right) \\
& \text { si } \alpha=\left(\alpha_{1}, \ldots, \alpha_{p}\right) \text { et } x_{1}, \ldots, x_{p} \text { dans } \mathbb{G} .
\end{aligned}
$$

Une $p$-cochaine non nulle sera dite homogène d'ordre $k$ si on a $c_{\alpha}\left(x_{1}, \ldots, x_{p}\right)=0$ pour tous les multi-indices $\alpha=\left(\alpha_{1}, \ldots, \alpha_{p}\right)$ tels que $|\alpha| \neq k$.

Le crochet étant d'ordre 0 , si $c$ est une cochaine d'ordre $k$, son bord est aussi une cochaine d'ordre $k$, donc l'espace des cochaines d'ordre $k$ de $\mathbb{G}_{\infty}(m)$ dans $\mathbb{G}$, noté $C^{*}\left(\mathbb{G}_{\infty}(m), \mathbb{G}\right)_{k}$, est un sous complexe du complexe de Chevalley-Eilenberg pour la représentation adjointe.

On a une décomposition en somme directe de sous complexes du complexe des cochaines de $\mathbb{G}_{\infty}(m)$ à valeurs dans $\mathbb{G}$ continues (pour la topologie $m$-adique de $\left.\mathbb{G}_{\infty}(m)\right) C_{c}^{*}\left(\mathbb{G}_{\infty}(m), \mathbb{G}\right)=\bigoplus_{k \geq 0} C_{c}^{*}\left(\mathbb{G}_{\infty}(m), \mathbb{G}\right)_{k}$.

Il y a donc une décomposition analogue au niveau de la cohomologie $H_{c}^{*}\left(\mathbb{G}_{\infty}(m), \mathbb{G}\right)=\bigoplus_{k \geq 0} H_{c}^{*}\left(\mathbb{G}_{\infty}(m), \mathbb{G}\right)_{k}$.

1.3. Position du problème. Le lien entre les deux cohomologies définies précédemment est donné par le théorème suivant:

Théorème 1.1 ([1, Proposition 1]). Soit $M$ une variété parallélisable, on $a$ :

$$
H_{\text {loc }}^{*}\left(\mathbb{G}_{F}, \mathbb{G}_{F}\right)=H_{c}^{*}\left(\mathbb{G}_{\infty}(m), \mathbb{G}\right) \otimes C^{\infty}(M ; \mathbb{C}) .
$$

Nous allons voir que le calcul de $H_{\text {loc }}^{2}\left(\mathbb{G}_{F}, \mathbb{G}_{F}\right)$ se ramène au calcul de la cohomologie des cochaines $\mathbb{G}$-invariantes continues de $\mathbb{G}_{\infty}(m)$ à valeur dans $\mathbb{G}$, ce calcul va être l'objet du paragraphe 3 .

Le paragraphe 2 est consacré à introduire le groupe de Poincaré et ses invariants que l'on va utiliser dans le paragraphe 3 . Dans la suite $P$ désignera l'algèbre de Lie du groupe de Poincaré que l'on apellera algèbre de Lie de Poincaré pour abréger.

On suppose que le fibré est trivial et que la variété est parallélisable, le passage au cas général se fait sans difficultés majeures (le calcul de la cohomologie de l'algèbre des courants devient un problème purement algébrique en remplaçant $\mathbb{G}_{F}$ par $\mathbb{G} \otimes \mathbb{G}_{\infty}(m)$ qui est le produit tensoriel de l'algèbre de Lie $\mathbb{G}$ par une algèbre lisse). 
Une grande partie de ce travail est consacrée aux calculs cohomologiques afin d'étudier les déformations de l'algèbre des courants; rappelons brièvement les définitions nécessaires.

\subsection{Déformation d'une algèbre de Lie.}

1.4.1. Crochet de Richardson-Nijenhuis [4]. A tout espace vectoriel $V$ on associe l'espace $\mathbb{A}(V)=\bigoplus_{p \geq-1} A^{P}(V)$ où $A^{P}(V)$ est l'espace des applications $(p+1)$ linéaires alternées de $V$ à valeurs dans $V$. Muni du crochet de Richardson-Nijenhuis $\mathbb{A}(V)$ est une algèbre de Lie graduée. Le crochet de Richardson-Nijenhuis est défini de la manière suivante:

Si $A \in A^{a}(V)$ et $B \in A^{b}(V)$ on définit le produit intérieur $i(B) \cdot A$, qui est une application $(p+q+1)$ linéaire de $V$ à valeur dans $V$, par $i(B) \cdot A\left(x_{0}, x_{1}, \ldots, x_{p+q}\right)=A\left(B\left(x_{0}, x_{1}, \ldots, x_{p}\right), x_{p+1}, \ldots, x_{p+q}\right)$ et on pose

$$
[|A, B|]=\frac{(a+b+1) !}{a !(b+1) !} \alpha(i(B) \cdot A)+(-1)^{a+b+1} \frac{(a+b+1) !}{b !(a+1) !} \alpha(i(A) \cdot B) .
$$

1.4.2. Soit $V$ un espace vectoriel et $P_{0}: V \times V \rightarrow V$ un crochet de Lie sur $V$, on notera $\mathbb{G}=\left(V, P_{0}\right)$ l'algèbre de Lie obtenue. Une déformation vraie à l'ordre 1 de $\mathbb{G}$ est une famille à un paramètre $P_{t}=P_{0}+t P_{1}$ d'applications bilinéaires antisymétriques de $V \times V$ dans $V$, telle que $P_{t}$ soit un crochet de Lie. Une famille $P_{t}=P_{0}+t P_{1}$ définit une déformation vraie à l'ordre 1 si et seulement si $\left[\left|P_{t}, P_{t}\right|\right]=0$. Comme $\left[\left|P_{0}, P_{0}\right|\right]=0$, cette condition équivaut à $\left[\left|P_{0}, P_{1}\right|\right]=0$ et $\left[\left|P_{1}, P_{1}\right|\right]=0$.

\section{Préliminaires sur le groupe de Poincaré et ses invariants}

2.1. Rappel sur l'algèbre de Lie so $(3,1)$. Posons

$$
J=\left[\begin{array}{llll}
-1 & & & 0 \\
& -1 & & \\
& & -1 & \\
0 & & & 1
\end{array}\right] ;
$$

on identifie alors so $(3,1)$ à $\left\{A \in M(4, \mathbb{R}) /{ }^{t} A J+J A=0\right\}$.

Nous utiliserons la base suivante de so(3,1) formée des matrices $M_{i}$, $N_{i}, 1 \leq i \leq 3$ telles que:

$$
\left[M_{i}, M_{j}\right]=\left[N_{j}, N_{i}\right]=\varepsilon_{i j k} M_{k} ;\left[M_{i}, N_{j}\right]=\varepsilon_{i j k} N_{k} .
$$

Pour $1 \leq i, j, k \leq 3 ;\left(\varepsilon_{i j k}\right)$ étant le tenseur complètement antisymétrique $\varepsilon_{123}=1$. 
Il y a un isomorphisme entre so $(3,1)$ et l'algèbre de Lie réelle $\operatorname{sl}(2, \mathbb{C})$ des matrices carrées d'ordre 2 à coefficients dans $\mathbb{C}$ de trace nulle. Soit $\left\{\sigma_{i}, i=1, \ldots, 4\right\}$ une base de l'espace vectoriel $H$ des matrices carrées hermitiennes d'ordre 2 définie par:

$$
\sigma_{1}=\left(\begin{array}{cc}
0 & 1 \\
1 & 0
\end{array}\right) ; \quad \sigma_{2}=\left(\begin{array}{cc}
0 & -i \\
i & 0
\end{array}\right) ; \quad \sigma_{3}=\left(\begin{array}{cc}
1 & 0 \\
0 & -1
\end{array}\right) ; \quad \sigma_{4}=\mathrm{id} .
$$

L'isomorphisme est donné par $M_{j} \rightarrow-\frac{i}{2} \sigma_{j} ; N_{j} \rightarrow \frac{1}{2} \sigma_{j} ; 1 \leq j \leq 3$.

Le groupe de Poincaré est le groupe des isométries affines de l'espace temps de Minkowski. Son algèbre de Lie $P$ s'identifie au produit semidirect de so $(3,1)$ par $\mathbb{R}^{4}$ via l'action naturelle de so(3,1) sur $\mathbb{R}^{4}$. En posant $L=\operatorname{so}(3,1)$ et $T=\mathbb{R}^{4}$, nous notons cette algèbre de Lie $P=$ $L \ltimes T$.

Pour $(a, b) \in\left(\mathbb{R}^{4}\right)^{2}$ et $A, B \in \operatorname{so}(3,1)$ on a donc le crochet:

$$
[(A, a),(B, b)]=([A, B], A b-B a) \text {, où }[A, B]=A B-B A .
$$

Dans la base précédente de so $(3,1)$ et la base canonique $\left(e_{i}\right)(i=$ $1,2,3,4)$ de $\mathbb{R}^{4}$, ce crochet s'exprime par:

$$
\begin{array}{rlrl}
{\left[M_{j}, e_{k}\right]} & =\varepsilon_{j k l} e_{l} ; & {\left[M_{j}, e_{4}\right]=0 ;} & \\
{\left[N_{j}, e_{j}\right]=e_{4} ;} & {\left[N_{j}, e_{4}\right]=e_{j} ; \quad 1 \leq j, k, l \leq 3 .}
\end{array}
$$

Nous pouvons aussi réaliser l'algèbre de Poincaré $P$ comme une algèbre de matrices; en effet $P$ s'identifie à l'espace des matrices $X \in M(3, \mathbb{C})$ telles que

$$
X=\left(\begin{array}{ccc}
a & b & u \\
c & -a & v \\
0 & 0 & 0
\end{array}\right)
$$

dont le crochet est défini par $\left[X_{1}, X_{2}\right]=X_{1} X_{2}-X_{2} X_{1}$.

La sous algèbre $P_{1}=\{X \in M(3, \mathbb{C}) / u=v=0\}$ est semi simple et correspond au sous-groupe des transformations homogènes (transformations de Lorentz).

L'idéal $P_{2}=\{X \in M(3, \mathbb{C}) / a=b=c=0\}$ correspond au sous groupe des translations.

2.2. Les applications bilinéaires $P$-invariantes. Nous allons déterminer les applications bilinéaires $P$-invariantes de $P$ à valeurs dans $P$, qui vont nous servir pour calculer $H_{c}^{2}\left(P_{\infty}(m), P\right.$ ) (Théorème 3.1 ) dans le paragraphe 3 .

Soit $s: P \times P \rightarrow P$ bilinéaire et $P$-invariante; $s$ vérifie $[X, s(Z, Y)]=$ $s([X, Z], Y)+s(Z,[X, Y])$ pour tous les $X, Y, Z$ dans $P$. 


\section{Proposition 2.1.}

i) Soit $s: L \times T \rightarrow P$ une application bilinéaire et $L$-invariante alors:

$$
\begin{aligned}
s\left(M_{i}, e_{j}\right) & =-s\left(M_{j}, e_{i}\right)=D\left[M_{i}, e_{j}\right] & & 1 \leq i<j \leq 3 \\
s\left(N_{i}, e_{i}\right) & =D e_{4} ; s\left(N_{i}, e_{4}\right)=D e_{i} & & 1 \leq i \leq 3 \text { où } D \text { est un réel. }
\end{aligned}
$$

ii) Soit c $: T \times T \rightarrow P$ une application bilinéaire et $P$-invariante alors il existe $\beta \in \mathbb{C}$ tel que $C\left(e_{i}, e_{j}\right)=\beta \varepsilon_{i j k} M_{k}$ et $C\left(e_{i}, e_{4}\right)=\beta N_{i}$, $1 \leq i<j<k \leq 3$.

Lemme 2.2. Soit s une forme bilinéaire sur $P$ telle que $s([X, Y], Z)+$ $s([X, Z], Y)=0$, pour tous $X, Y, Z$ dans $P$.

Alors si $B$ est une forme de Killing sur $\operatorname{sl}(2, \mathbb{C})$, il existe $\lambda \in \mathbb{R}$ tel que:

i) $s\left(z, z^{\prime}\right)=\lambda B\left(z, z^{\prime}\right)$ pour $\left(z, z^{\prime}\right) \in L \times L$.

ii) $s(X, Y)=0$ pour $(X, Y) \in T \times L$.

iii) $s\left(e_{i}, e_{j}\right)=0$ si $i \neq j$ et $1 \leq j, i \leq 3 ; s\left(e_{i}, e_{i}\right)=-s\left(e_{4}, e_{4}\right)=0$ pour $1 \leq i \leq 3$ où $e_{i} ; i=1, \ldots, 4$ est une base de $T$. On note par la suite une telle forme $\sigma$.

\section{Le calcul de $H^{*}\left(P_{F}, P_{F}\right)$}

Pour calculer $H^{*}\left(P_{F}, P_{F}\right)$ on se ramène à $H^{*}\left(P_{\infty}(m), P\right)$ (cf. Théorème 1.1) que l'on calcule en utilisant la suite spectrale de HochschildSerre. Soit $\bar{P}_{\infty}(m)$ l'idéal de $P_{\infty}(m)$ formé des polynômes sans terme constant; alors $P_{\infty}(m) / \bar{P}_{\infty}(m)$ est isomorphe à $P$, d'où la suite exacte courte d'algèbres de Lie $0 \rightarrow \bar{P}_{\infty}(m) \rightarrow P_{\infty}(m) \rightarrow P \rightarrow 0$.

Nous considérons la suite spectrale de Hochschild-Serre associée à cette suite exacte. Le terme de $E_{2}$ de cette suite spectrale est donné par: $E_{2}^{p, q}=H^{P}\left(P, H^{q}\left(\bar{P}_{\infty}(m), P\right)\right)$. Le terme $E_{\infty}$ de cette suite spectrale est associé à une filtration de $H^{*}\left(P_{\infty}(m), P\right)$. On va calculer $H_{c}^{*}\left(P_{\infty}(m), P\right)$.

Théorème 3.1. $H_{c}^{2}\left(P_{\infty}(m), P\right)=H^{2}(P, P) \oplus \operatorname{Inv}_{L} H_{c}^{2}\left(\bar{P}_{\infty}(m), P\right)$, où $\operatorname{Inv}_{L} H_{c}^{2}\left(\bar{P}_{\infty}(m), P\right)$ désigne l'espace des classes de cochaines invariantes par l'action de $L$.

Pour la démonstration de ce théorème on utilise les Propositions 3.5, 3.6, 3.7 et 3.8 .

Rappelons d'abord quelques théorèmes utiles par la suite. Dans certains cas, la suite spectrale de Hochschild-Serre dégénère et on a: 
Théorème 3.2 (G. Hochschild-J. P. Serre [2]). Soit $g$ une algèbre de Lie de dimension finie sur un corps $K$ de caractéristique 0 et soit $M$ un g-module de dimension finie et soit $T$ un idéal abélien tel que $g / T$ soit semi simple. Alor pour $n \geq 0$ on a

$$
H^{n}(g, M) \approx \sum_{i+j=n} H^{j}(g / T, K) \otimes \operatorname{Inv}_{g} H^{i}(T, M),
$$

où $\operatorname{Inv}_{g} H^{i}(T, M)$ est l'ensemble des éléments de $H^{i}(T, M)$ invariants par $g$.

Lemme 3.3 (Whitehead [3]). Soit g une algèbre de Lie de dimension finie et $A$ un $g$-module de dimension finie; si $g$ est semi simple alors $H^{1}(g, A)=0$.

Lemme 3.4. $H^{1}(P, \operatorname{Hom}(P, P))=0$.

Démonstration: Nous allons encore utiliser le théorème de Hochschild et Serre: $H^{1}(P, \operatorname{Hom}(P, P)) \approx H^{0}(P / T, \mathbb{R}) \otimes \operatorname{Inv}_{P} H^{1}(T, \operatorname{Hom}(P, P))=$ $\operatorname{Inv}_{P} H^{1}(T, \operatorname{Hom}(P, P))$. $L$ est semi simple alors cet space de cohomologie est égal à la cohomologie des cochaines $\operatorname{Inv}_{L} C^{1}(T, \operatorname{Hom}(P, P))$. On identifie $C^{1}(T, \operatorname{Hom}(P, P))$ à l'espace des applications bilinéaires de $T \times P$ à valeurs dans $P$. La condition d'invariance se traduit par $M \cdot C(X)=$ $C([M, X])$ pour tout $M \in L, X \in T$, d'autre part $M \cdot C(X) Y=$ $[M, C(X) Y]-C(X)([M, Y])$, d'où

$$
[M, C(X)](Y)-C([M, X]) Y-C(X)([M, Y])=0
$$

pour tout $X \in T, Y \in P$ et $M \in P$. D'après la Proposition 2.1, les cochaines $s L$-invariantes de $T$ dans $\operatorname{Hom}(P, P)$ sont définies par:

$$
\begin{aligned}
& s\left(M_{i}, e_{j}\right)=-s\left(M_{j}, e_{i}\right)=D\left[M_{i}, e_{j}\right], \quad 1 \leq i<j \leq 3 \\
& s\left(N_{i}, e_{4}\right)=D e_{i} ; s\left(N_{i}, e_{4}\right)=D e_{i}, \quad 1 \leq i \leq 3 \text { où } D \text { est un réel. }
\end{aligned}
$$

Mais $s$ est un cobord dans $C^{1}(T, \operatorname{Hom}(P, P))$; en effet $s$ est une dérivation intérieure, $s=\operatorname{ad} \tau$ avec $\tau \in \operatorname{Hom}(P, P)$ défini par:

$$
\begin{array}{cl}
\tau\left(M_{i}\right)=D M_{i} & 1 \leq i \leq 3 \text { et } \tau / T=0 \\
\tau\left(N_{i}\right)=D N_{i} & 1 \leq i \leq 3 .
\end{array}
$$

D'autre part si $C: T \times T \rightarrow P$ est bilinéaire $P$-invariante alors

$$
\begin{aligned}
& C\left(e_{i}, e_{j}\right)=\beta \varepsilon_{i j k} M_{k}, \quad \beta \in \mathbb{C}, 1 \leq i, j \leq 3 \\
& C\left(e_{i}, e_{4}\right)=\beta N_{i} .
\end{aligned}
$$

En identifiant $C$ à une cochaine dans $C^{1}(T, \operatorname{Hom}(P, P))$, notée encore $C$, la condition de cocycle se traduit par:

$$
[X, C(Y) Z]-[Y, C(X) Y]+C(X)[Y, Z]-C(Y)[X, Z]=0
$$


pour tous $X, Y \in T$ et $Z \in P$; d'où

$$
\begin{aligned}
{\left[e_{i}, C\left(e_{j}, M_{i}\right)\right]-\left[e_{j}, C\left(e_{j}, M_{i}\right)\right] } & +\left[e_{j}, C\left(e_{i}, M_{i}\right)\right] \\
& -C\left(e_{i},\left[e_{j}, M_{i}\right]\right)-C\left(e_{j},\left[e_{i}, M_{i}\right]\right)=0,
\end{aligned}
$$

et

$\left[e_{i}, c\left(e_{j}, N_{i}\right)\right]-\left[e_{j}, C\left(e_{i}, N_{i}\right)\right]+C\left(e_{i},\left[e_{j}, N_{i}\right]\right)-C\left(e_{j},\left[e_{i}, N_{i}\right]\right)=0$.

On obtient donc $c\left(e_{i}, e_{j}\right)=0,1 \leq i, j \leq 3$ et $c\left(e_{j}, e_{4}\right)=0,1 \leq i \leq 3$. Ce qui achève la démonstration du Lemme 3.4.

Proposition 3.5. $E_{2}^{2,0}=H^{2}\left(P, H_{c}^{0}\left(\bar{P}_{\infty}(m), P\right)\right)$ est de dimension 1 .

Démonstration: L'action de $\bar{P}_{\infty}(m)$ sur $P$ étant triviale, on a $H^{0}\left(\bar{P}_{\infty}(m), P\right)=P$, donc $H^{2}\left(P, H_{c}^{0}\left(\bar{P}_{\infty}(m), P\right)\right)=H^{2}(P, P)$; or d'après un théorème de Levy-Nahas [6] on a $\operatorname{dim} H^{2}(P, P)=1$. Par conséquent, $H^{2}(P, P)$ est engendré par un 2-cocycle $C$ qui vérifie:

$$
C\left(e i, e_{j}\right)=\varepsilon_{i j k} M_{k} ; C\left(e i, e_{4}\right)=N_{i} \text { pour } 1 \leq i, j, k \leq 3 ;
$$

d'où le résultat.

Proposition 3.6. $E_{2}^{1,1}=H^{1}\left(P, H_{c}^{1}\left(\bar{P}_{\infty}(m), P\right)\right)=0$.

Démonstration: L'action de $\bar{P}_{\infty}(m)$ sur $P$ est triviale donc:

$H_{c}^{1}\left(\bar{P}_{\infty}(m), P\right)=\operatorname{Hom}\left(H_{1}\left(\bar{P}_{\infty}(m)\right), P\right)=\operatorname{Hom}\left(\frac{\bar{P}_{\infty}(m)}{\left[\bar{P}_{\infty}(m), \bar{P}_{\infty}(m)\right]}, P\right)$.

Le commutateur $\left[\bar{P}_{\infty}(m), \bar{P}_{\infty}(m)\right]$ est l'ensemble des éléments du type $\sum_{\alpha} t^{\alpha} x_{\alpha}$ où $x_{\alpha} \in P$ avec $|\alpha|>1$ où $\alpha \in \mathbb{N}^{m}$ un multi-indice. On en déduit donc que $H_{1}\left(\bar{P}_{\infty}(m)\right)$ s'identifie à $P \otimes \mathbb{R}^{m}$ en considérant tous les multiindices $\alpha$ tels que $|\alpha|=1$, et donc $H_{c}^{1}\left(\bar{P}_{\infty}(m), P\right)=\operatorname{Hom}(P, P) \otimes$ $\mathbb{R}^{m}$.

D'après le Lemme 3.4 on a $H^{1}(P, \operatorname{Hom}(P, P))=0$ d'où

$$
H^{1}\left(P, H_{c}^{1}\left(\bar{P}_{\infty}(m), P\right)\right)=0 .
$$

Ce qui achève la démonstration de la Proposition 3.6.

Pour obtenir le $H^{2}$, il nous reste à déterminer le terme $E_{2}^{0,2}$. Il va être déterminé en deux étapes: dans la Proposition 3.7 on détermine $H^{0}\left(P, H^{2}\left(\bar{P}_{\infty}(m), P\right)\right)_{k}$ pour $k>2$ et dans la Proposition 3.8 on détermine $H^{0}\left(P, H^{2}\left(\bar{P}_{\infty}(m), P\right)\right)_{2}$.

Proposition 3.7. $H^{0}\left(P, H^{2}\left(\bar{P}_{\infty}(m), P\right)\right)_{k}=\operatorname{Inv}_{L} H^{2}\left(\bar{P}_{\infty}(m), P\right)_{k}=0$ pour $k>2$. 
Démonstration: Pour des raisons de semi-simplicité, $\operatorname{Inv}_{L} H^{2}\left(\bar{P}_{\infty}(m), P\right)_{k}$ est égale à la cohomologie des cochaines $L$-invariantes $C^{2}\left(\bar{P}_{\infty}(m), P\right)_{k}$.

Soit

$$
I_{k}^{p}=\left\{\left(\alpha_{0}, \ldots, \alpha_{p-1}\right) ; \alpha_{i} \in \mathbb{N}^{m} ; \sum_{i}\left|\alpha_{i}\right|=k\right\}
$$

et soit $C \in C^{2}\left(\bar{P}_{\infty}(m), P\right)_{k}$ un 2-cocycle; la condition de cocycle s'écrit:

$$
\begin{array}{r}
C\left(\left[x_{1}, x_{2}\right], x_{3}\right)+C\left(\left[x_{2}, x_{3}\right], x_{1}\right)+C\left(\left[x_{3}, x_{1}\right], x_{2}\right)=0 \\
\text { pour } x_{1}, x_{2}, x_{3} \text { dans } P .
\end{array}
$$

Soit $C_{\alpha, \beta} \in I_{k}^{2}$ les composantes du 2 cocyle $C$. La condition précédente de cocycle implique:

$$
C_{\alpha, \beta+\gamma}\left(x_{1},\left[x_{2}, x_{3}\right]\right)+C_{\gamma, \alpha+\beta}\left(x_{3},\left[x_{1}, x_{2}\right]\right)+C_{\alpha+\gamma, \beta}\left(\left[x_{1}, x_{3}\right], x_{2}\right)=0 .
$$

Soit $s=C_{\alpha+\beta, \gamma}-C_{\alpha+\gamma, \beta}$.

On a

$$
\begin{aligned}
& s\left(\left[x_{1}, x_{2}\right], x_{3}\right)=C_{\alpha+\beta, \gamma}\left(\left[x_{1}, x_{2}\right], x_{3}\right)-C_{\alpha+\gamma, \beta}\left(\left[x_{1}, x_{2}\right], x_{3}\right), \quad \text { et } \\
& s\left(\left[x_{1}, x_{3}\right], x_{2}\right)=C_{\alpha+\beta, \gamma}\left(\left[x_{1}, x_{3}\right], x_{2}\right)-C_{\alpha+\gamma, \beta}\left(\left[x_{1}, x_{3}\right], x_{2}\right) .
\end{aligned}
$$

Or la condition du cocycle nous donne:

$$
\begin{aligned}
& C_{\alpha, \beta+\gamma}\left(x_{1},\left[x_{2}, x_{3}\right]\right)=C_{\alpha+\beta, \gamma}\left(\left[x_{1}, x_{2}\right], x_{3}\right)-C_{\alpha+\gamma, \beta}\left(\left[x_{1}, x_{3}\right], x_{2}\right) \quad \text { et } \\
& C_{\alpha, \beta+\gamma}\left(x_{1},\left[x_{3}, x_{2}\right]\right)=C_{\alpha+\beta, \gamma}\left(\left[x_{1}, x_{3}\right], x_{2}\right)-C_{\alpha+\gamma, \beta}\left(\left[x_{1}, x_{2}\right], x_{3}\right)
\end{aligned}
$$

donc finalement $s\left(\left[x_{1}, x_{2}\right], x_{3}\right)+s\left(\left[x_{1}, x_{3}\right], x_{2}\right)=0$.

Donc $s$ vérifie la condition du Lemme 2.2; $s$ est alors le produit de la forme $\sigma$ sur $L$ par un élément $v$ de $P$ fixé. Mais $s$ est $L$ invariant, $v$ est $L$ invariant. On obtient donc $v=0, s=0$ et $C_{\alpha+\beta, \gamma}=C_{\beta+\gamma, \alpha}=C_{\gamma+\alpha, \beta}$ pour tous les $(\alpha, \beta, \gamma)$ de $I_{k}^{3}$.

Soit $\varepsilon=C_{\alpha, \beta}-C_{\beta, \alpha} ; \varepsilon$ est $L$-invariante. D'autre part $\varepsilon$ est antisymétrique donc $\varepsilon=0$ c'est à dire $C_{\alpha, \beta}=C_{\beta, \alpha}$. Donc pour $k>2$ il existe une application: $b: I_{k}^{1} \rightarrow P^{*} \otimes P^{*} \otimes P$ telle que $C_{\alpha, \beta}=b_{\alpha+\beta}$ pour $(\alpha, \beta) \in I_{k}^{2}$. $C$ étant un cocycle $L$-invariant alors pout tout $\alpha \in I_{k}^{1}$, $b_{\alpha}$ est un cocycle $P$-invariant pour la représentation triviale de $P$ dans $P$. On notera $H^{*}\left(P, P_{\text {triv }}\right)$ la cohomologie correspondante pour éviter les confusions avec $H^{*}(P, P)$. 
On a $H^{2}\left(P, P_{\text {triv }}\right) \approx H^{2}(P, \mathbb{R}) \otimes P$. D'après $\left[\mathbf{5}\right.$, p. 252] $H^{2}(P, \mathbb{R})$ est de dimension 1 engendré par la classe de cohomologie du cocycle

$$
\begin{gathered}
C\left(X_{1}, X_{2}\right)=\operatorname{Re} \lambda\left(u_{1} v_{2}-v_{1} u_{2}\right), \lambda \in \mathbb{C}, \\
X_{1}=\left(\begin{array}{ccc}
0 & 0 & u_{1} \\
0 & 0 & v_{1} \\
0 & 0 & 0
\end{array}\right), X_{2}=\left(\begin{array}{ccc}
0 & 0 & u_{2} \\
0 & 0 & v_{2} \\
0 & 0 & 0
\end{array}\right) \in P_{2} ;
\end{gathered}
$$

on utilise ici la réalisation de l'algèbre de Poincaré comme étant l'espace des matrices $X \in M(3, \mathbb{C})$ telles que

$$
X=\left(\begin{array}{ccc}
a & b & u \\
c & -a & v \\
0 & 0 & 0
\end{array}\right)
$$

(voir page 4 pour les notations).

Maintenant on montre que $\operatorname{Inv}_{P} H^{2}\left(P, P_{\text {triv }}\right)=0$; en effet pour $C$ dans $H^{2}(P, \mathbb{R}), X$ dans $P$, on note $C_{X}=C \otimes X$, c'est un cocycle pour la représentation triviale de $P$ dans $P$. La condition de $P$-invariance se traduit par: $\left[Y, C_{X}\left(X_{1}, X_{2}\right)\right]=C_{X}\left(\left[Y, X_{1}\right], X_{2}\right)+C_{X}\left(X_{1},\left[Y, X_{2}\right]\right)$ pour tout $Y$ dans $P$, on a alors $X=0$ d'où le résultat.

Proposition 3.8. $H^{0}\left(P, H^{2}\left(\bar{P}_{\infty}(m), P\right)\right)_{2}$, étant identifié à $\operatorname{Inv}_{L} H^{2}\left(\bar{P}_{\infty}(m), P\right)$, est engendré par une classe de cohomologie représentée par un cocycle $C$ dont les composantes sont définies par $C_{\alpha, \beta}\left(e_{i}, e_{j}\right)=u^{\alpha \beta} C\left(e_{i}, e_{j}\right)$ où $u^{\alpha \beta} \in \mathbb{R} ; u^{\alpha \beta}=u^{\beta \alpha}$ et $C\left(e_{i}, e_{j}\right)=$ $\gamma \varepsilon_{i j k} M_{k} ; C\left(e i, e_{4}\right)=\gamma N_{i}, 1 \leq i \leq j \leq 3, \gamma \in \mathbb{C}$.

Démonstration: D'après la Proposition 2.4 les cochaines $L$-invariante de $\bar{P}_{\infty}(m)$ à valeur dans $P$ sont définies par leurs composantes homogènes de la façon suivante:

i) Pour $X \in L$ et $Y \in T, s_{\alpha, \beta}(X, Y)=v^{\alpha \beta} s(X, Y), v^{\alpha \beta} \in \mathbb{R}$;

$$
\begin{array}{ll}
s\left(M_{i}, e_{j}\right)=-s\left(M_{j}, e_{i}\right)=D\left[M_{i}, e_{j}\right], & \text { pour } D \in \mathbb{R}, \quad 1 \leq i<j \leq 3 \\
s\left(N_{i}, e_{4}\right)=D e_{i} ; s\left(N_{i}, e_{i}\right)=D e_{4}, & \text { pour } D \in \mathbb{R}, \quad 1 \leq i \leq 3 .
\end{array}
$$

$\left(v^{\alpha \beta}\right)$ est un 2-tenseur symétrique, on vérifie facilement que $s$ est un cobord.

ii) $C_{\alpha, \beta}=u^{\alpha \beta} C\left(e_{i}, e_{j}\right)$ où $u^{\alpha \beta} \in \mathbb{R}$ et $C\left(e_{i}, e_{j}\right)=\beta \varepsilon_{i j k} M_{k} ; C\left(e_{i}, e_{4}\right)=$ $\beta N_{i}, 1 \leq i, j \leq 3, \beta \in \mathbb{C}$.

La cochaine $C$ étant anti-symétrique, on lui associe un tenseur symétrique $\left(u^{\alpha \beta}\right) \in \Lambda^{2} \mathbb{R}^{m}$, d'où $H^{0}\left(P, H^{2}\left(\bar{P}_{\infty}(m), P\right)\right)_{2}$ est isomorphe à $S^{2} \mathbb{R}^{m}$. Ce qui achève la démonstration de la Proposition 3.8. 
Maintenant on calcule la suite spectrale de Hochschild et Serre et on montre qu'elle dégénère au terme d'ordre 2 ; pour cela on démontre le lemme suivant:

Lemme 3.9. $E_{2}^{2,1}=H^{2}\left(P, H_{c}^{1}\left(\bar{P}_{\infty}(m), P\right)\right)=0$.

Démonstration: Nous avons vu dans la démonstration de la Proposition 3.5 que $H_{c}^{1}\left(\bar{P}_{\infty}(m), P\right)=\operatorname{Hom}(P, P) \otimes \mathbb{R}^{m}$ et d'après le lemme de Whitehead on a $H^{2}\left(P, H_{c}^{1}\left(\bar{P}_{\infty}(m), P\right)\right)=\operatorname{Inv}_{P} C^{2}\left(T, \operatorname{Hom}(P, P) \otimes \mathbb{R}^{m}\right)$.

Soit $C$ une 2-cochaine $P$-invariante de $T$ à valeurs dans $\operatorname{Hom}(P, P)$; la condition de $P$-invariance se traduit par: $[M, C(x, y)]=C([M, x], y)+$ $C(x,[M, y])$ pour tous les $x$ et $y$ dans $T$ et $M$ dans $P$. Or pour $z \in T$, $[M, C(x, y)](z)=[M, C(x, y)(z)]-C(x, y)([M, z])$.

Par conséquent $\tilde{C}: T^{3} \rightarrow P$ définie par $\tilde{C}(x, y, z)=C(x, y)(z)$ est $L$-invariant or $\operatorname{Inv}_{L} C^{3}(T, P)=0([\mathbf{6}])$ d'où le résultat.

Revenons maintenant à la suite spectrale de Hochschild et Serre, on sait que $E_{2}^{1,1}=0$, donc $E_{\infty}^{1,1}=0$. D'autre part d'après le Lemme 3.9, $E_{2}^{2,1}=0$ donc $E_{2}^{0,2}=E_{\infty}^{0,2}$ et la suite spectrale dégénère au terme d'ordre 2. Finalement on obtient le résultat du Théorème 3.1:

$$
H_{c}^{2}\left(P_{\infty}(m), P\right)=H^{2}(P, P) \oplus \operatorname{Inv}_{L} H_{c}^{2}\left(\bar{P}_{\infty}(m), P\right) .
$$

Revenons maintenant à la cohomologie locale de l'algèbre des courants. La correspondance entre les deux cohomologies (cf. Théorème 1.1) peut se voir au niveau des cochaines:

$$
\begin{aligned}
I: \quad C^{p}\left(P_{\infty}(m), P\right) \otimes C^{\infty}(M) & \longrightarrow C^{p}\left(P_{F}, P_{F}\right) \\
(s \otimes a) & \longrightarrow I(s \otimes a)
\end{aligned}
$$

telle que $I(s \otimes a)\left(f_{1}, \ldots, f_{p}\right)(x)=a(x) \cdot s\left(j_{\infty} f_{1}(x), \ldots, j_{\infty} f_{p}(x)\right)$.

Proposition 3.10. Le deuxième espace de cohomologie locale de l'algèbre des courants associé à l'algèbre de Poincaré est $H_{\mathrm{loc}}^{2}\left(P_{F}, P_{F}\right)=$ $H^{2}(P, P) \otimes C^{\infty}(M) \otimes S^{2}(M)$; où $S^{2} M$ l'espace des 2-tenseurs contravariants symétriques.

Démonstration: Soit $s$ un 2-cocyle de $P$ à valeurs dans $P$ et $a$ dans $C^{\infty}(M)$, on assacie à $s \otimes a$ un 2-cocycle de $P_{F}$ à valeur dans $P_{F}$.

D'autre part soit $S^{2} M$ l'espace des 2-tenseurs contravariants symétriques, et $S \in S^{2}(M)$; on lui associe une unique application bilinéaire $C_{S}: P_{F} \times P_{F} \rightarrow P_{F}$ telle que, sur toute carte $\left(U ;\left(x_{1}, \ldots, x_{m}\right)\right)$ de $M$ où $S$ s'écrit, $S / U=\sum_{\alpha, \beta} S^{\alpha, \beta} \partial_{\alpha} \otimes \partial_{\beta}$ on ait

$$
C_{S}(f, g) / U=\sum_{\alpha, \beta} S^{\alpha, \beta} C\left(\partial_{\alpha} f(x), \partial_{\beta} g(x)\right),
$$


$C$ est le cocycle défini dans la Proposition 3.8. Si $f$ ou $g$ est dans $C^{\infty}(M, L)$ alors $C_{S}(f, g)=0$ car $C$ est identiquement nul si l'un des termes est à valeurs dans $L$.

De plus $C_{S}$ est un cocycle: en effet

$$
\partial C_{S}(f, g, h)(x)=\sum_{c y c l} S^{\alpha, \beta} C\left(\partial_{\alpha}[f(x), g(x)], \partial_{\beta} h(x)\right)
$$

or $[f(x), g(x)]=0$ car $T$ est abélien, d'où le résultat.

\section{Applications aux déformations}

Nous allons étudier d'abord les déformations correspondant au terme $\mathbb{R}$ provenant de $H^{2}(P, P)$.

On a vu que $H^{2}(P, P)$ est de dimension 1 engendré par une classe de cohomologie représentée par le cocycle $S$ définie par:

$C\left(e_{i}, e_{j}\right)=\varepsilon_{i j k} M_{k}$ pour $1 \leq i \leq j \leq k \leq 3$ et $C\left(e_{i}, e_{4}\right)=N_{i}$ pour $1 \leq i \leq 3$. Ce cocycle permet de définir la déformation de $P$ vers l'algèbre de Lie du groupe de De Sitter so $(4,2)$.

Pour l'algèbre des courants $P_{F}$, on a la déformation définie par: $[f(x), g(x)]_{t}=[f(x), g(x)]+t C(f(x), g(x))$ pour $f, g \in C^{\infty}(M, P)$ et $x \in M$.

Comme $H^{2}(P, P)$ est inclu dans $H_{\mathrm{loc}}^{2}\left(P_{F}, P_{F}\right)$, les déformations de $P$ peuvent être réalisées comme déformations d'ordre 0 de $P_{F}$.

Etudions maintenant les déformations correspondant aux tenseurs de $S^{2}(M)$.

Soit $C_{S}$ le cocycle déterminé par un 2-tenseur arbitraire $S$ de $S^{2}(M)$, nous allons montrer la nullité du crochet de Richardson-Nijenhuis $\left[\left|C_{S}, C_{S}\right|\right]$.

(*)

$$
\begin{aligned}
{\left[\mid C_{S}, C_{S}\right](f, g, h) } & =2 \sum_{c y c l} C_{S}\left(\sum_{\alpha, \beta} S^{\alpha \beta} C\left(\partial_{\alpha} f, \partial_{\beta} g\right), h\right) \\
& =2 \sum_{c y c l}\left(\sum_{\gamma, \mu} S^{\gamma \mu} C\left(\sum_{\alpha, \beta} \partial_{\gamma}\left(S^{\alpha \beta} C\left(\partial_{\alpha} f, \partial_{\beta} g\right), \partial_{\mu} h\right)\right)\right)
\end{aligned}
$$

or

$$
\begin{array}{r}
(* *) \quad S\left(\partial_{\alpha} f, \partial_{\beta} g\right)=\sum_{1 \leq k<1<m \leq 3}\left(\partial_{\alpha} f_{k}, \partial_{\beta} g_{l}-\partial_{\alpha} f_{l} \partial_{\beta} g_{k}\right) \varepsilon_{k l m} M_{m} \\
+\sum_{k=1}^{3}\left(\partial_{\alpha} f_{k} \partial_{\beta} g_{4}-\partial_{\alpha} f_{4} \partial_{\beta} f_{k}\right) N_{k} .
\end{array}
$$


En remplaçant $(* *)$ dans $(*), C\left(\partial_{\alpha} f, \partial_{\beta} g\right)$ est une fonction à valeurs dans $L$. Comme $C$ est identiquement nul si l'un des termes est dans $L$, on aura le nullité de chaque terme du type $C_{S}\left(\sum_{\alpha, \beta} S^{\alpha \beta} C\left(\partial_{\alpha} f, \partial_{\beta} g\right), h\right)$ d'où $\left[\left|C_{S}, C_{S}\right|\right]=0$.

Tout cocycle $C_{S}$ définit ainsi une déformation vraie à l'ordre 1 de l'algèbre des courants $P_{F}$

$$
\begin{array}{r}
{[f(x), g(x)]_{t}=[f(x), g(x)]+t C_{S}(f(x), g(x))} \\
\quad \text { pour } f, g \in C^{\infty}(M, P) \text { et } x \in M .
\end{array}
$$

Remerciement. Je remercie le professeur Claude Roger pour ses conversations fructueuses.

\section{Bibliographie}

[1] P. B. A. Lecomte et C. Roger, Rigidity of current Lie algebras of complex simple type, J. London Math. Soc. (2) 37(2) (1988), 232-240.

[2] G. Hochschild et J. P. Serre, Cohomology of Lie algebras, Ann. of Math. (2) 57 (1953), 591-603.

[3] P. J. Hilton et U. Stammbach, "A course in homological algebra", Graduate Texts in Mathematics 4, Springer-Verlag, New YorkBerlin, 1971.

[4] A. Nijenhuis et R. W. Richardson, JR., Deformations of Lie algebra structures, J. Math. Mech. 17 (1967), 89-105.

[5] A. Kirillov, "Éléments de la théorie des représentations", Éditions Mir, Moscow, 1974.

[6] M. Levy-Nahas, Deformation and contraction of Lie algebras, $J$. Mathematical Phys. 8 (1967), 1211-1222.

Département de Mathématiques

Faculté des Sciences

Université de Sfax

3038 Sfax

Tunisie

E-mail address: Faouzi.Ammar@fss.rnu.tn 